Images du travail, travail des images

$4 \mid 2017$

La relation soignants/soignés à l'épreuve de l'image

\title{
Grand entretien avec Thomas Lilti
}

Nadine Michau, Lucia Candelise, Gilles Remillet et Thomas Lilti

\section{OpenEdition}

\section{Journals}

Édition électronique

URL : http://journals.openedition.org/itti/1028

DOI : 10.4000/itti.1028

\section{Éditeur}

Université de Poitiers

\section{Référence électronique}

Nadine Michau, Lucia Candelise, Gilles Remillet et Thomas Lilti, « Grand entretien avec Thomas Lilti », Images du travail, travail des images [En ligne], 4 | 2017, mis en ligne le 01 septembre 2017, consulté le 14 avril 2021. URL : http://journals.openedition.org/itti/1028 ; DOI : https://doi.org/10.4000/itti.1028

Ce document a été généré automatiquement le 14 avril 2021

Images du travail, travail des images 


\title{
Grand entretien avec Thomas Lilti
}

\author{
Nadine Michau, Lucia Candelise, Gilles Remillet et Thomas Lilti
}

1 Thomas Lilti est né le 30 mai 1976, il mène ses activités de réalisateur et de scénariste en parallèle de ses études en médecine générale. Entre 1999 et 2004, Il réalise trois courts métrages et travaille comme scénariste pour huit films de fiction, c'est avec ses deux derniers longs métrages tournés sur le travail en milieu médical, Hippocrate en 2014 et Médecin de campagne en 2016, que son cinéma est plébiscité par le grand public. $\mathrm{Au}$ cours de cet entretien, Thomas Lilti revient sur son parcours de jeune médecin, sur les conditions d'exercice de ce métier à l'hôpital comme en milieu libéral tout en portant un regard critique sur les études de médecine qu'il a suivies. Toujours un peu en marge et en décalage par rapport aux attentes professionnelles et familiales de son milieu, jamais complètement médecin mais déjà toujours cinéaste, c'est à partir de cet entre-deux qu'il forgera son regard pour filmer à bonne distance et mettre à l'épreuve du cinéma son expérience personnelle du système de soin actuel.

2 La relation soignants-soignés en milieu hospitalier, traitée dans le film Hippocrate est pour lui souvent prétexte à installer des scènes intimes révélant les sentiments profonds des personnages, des moments où l'on accède à une autre réalité, une autre ambiance, dévoilant par exemple les coulisses, les normes et les codes des relations hiérarchiques interprofessionnelles entre médecins, internes, infirmières et aidessoignants. Thomas Lilti revient également sur ses influences cinématographiques qui semblent moins marquées par le documentaire que par les images de reportage télévisuel qui laissent selon lui bien plus apparaitre certaines ambiances, des éléments de décor, des attitudes, des gestes, véritables sources d'inspiration et de possibles projections pour son travail d'écriture cinématographique.

Dans Médecin de campagne, l'auteur dresse le portrait de l'exercice d'une médecine en contexte rural et d'un médecin confronté à sa propre maladie. Thomas Lilti rappelle dans l'entretien les moments marquants, les points de bascule dans le récit marqué par des transformations physiques et mentales d'un personnage à la fois soignant et soigné. Dans ces films de fiction, les relations de soin se dévoilent comme des scènes sociales prétextes à des réflexions sur la vie, la mort, l'assistance sociale de la maladie, les frontières entre le normal et le pathologique. Sont ainsi abordées les relations tissées 
entre science, santé et société : la complexité des relations de soins, des gestes médicaux et des dispositifs thérapeutiques met finalement le spectateur à l'épreuve de ses propres représentations sociales et culturelles, de la santé, de la médecine et de la maladie.

Ce média ne peut être affiché ici. Veuillez vous reporter à l'édition en ligne http://

\section{BIBLIOGRAPHIE}

\section{Filmographie}

\section{Comme réalisateur}

Quelques heures en hiver, 1999

Après l'enfance, 2002

Roue libre, 2004

Les Yeux bandés, 2007

Hippocrate, 2014

Médecin de campagne, 2016

\section{Comme scénariste}

Les Yeux bandés, 2007

Cour Océan, série TV, saisons 4 et 5, 2009

Mariage à Mendoza, 2011

Télé Gaucho, 2011

Gauguin d'Edouard Deluc (co-scénariste), 2017

Première Année, 2017

\section{AUTEURS}

\section{NADINE MICHAU}

Nadine Michau, anthropologue et cinéaste, réalise depuis plusieurs années des films documentaires ou sociologiques. Elle est actuellement chercheure associée au sein du laboratoire CITERES (UMR-CNRS) à l'Université François-Rabelais, où elle enseigne la réalisation 
de films documentaires aux étudiants du département de sociologie.

Elle a participé à de nombreuses recherches en utilisant le film comme outil d'investigation central. Ses objets de recherches sont principalement liés à la sociologie du travail : d'abord une anthropologie filmée des soins esthétiques en milieu professionnel, elle a ensuite réalisé des recueils audiovisuels portant sur la mémoire ouvrière industrielle, et vient d'achever un film documentaire sur la profession d'agriculteur. Elle poursuit ses recherches sur la place de l'image dans les sciences sociales.

\section{LUCIA CANDELISE}

Lucia Candelise a été la collaboratrice scientifique principale et maitre assistante à l'Université de Genève dans le cadre du projet : "Circulation, transmission et adaptation des pratiques médicales chinoises en Europe. Leur réception en Suisse pour une histoire comparée avec le contexte médical français et italien ", FNS 146539, entre septembre 2013 et décembre 2016. Elle mène depuis plusieurs années des recherches sur la diffusion, la réception et les tentatives d'intégration de la médecine chinoise dans les différents pays de l'Europe et d'Afrique en combinant à l'approche historique des enquêtes de terrain. Elle s'intéresse aussi à la patrimonialisation des savoirs médicaux. Elle est associée au laboratoire CECMC, UMR 8173, Chine, Corée, Japon (EHESS/CNRS), rattachée au laboratoire SPHERE, UMR 7219, CNRS/Paris 7, membre (chercheuse libre) à l'Institut universitaire d'histoire de la médecine et de la santé publique (IUHMSP-CHUV), Lausanne, et au laboratoire CETCOPRA Paris 1 la Sorbonne.

\section{GILLES REMILLET}

Gilles Remillet est anthropologue-cinéaste et membre de l'équipe de recherche Histoire des arts et des représentations (H.A.R) de l'Université Paris Ouest Nanterre la Défense, membre du CA du Comité du film ethnographique, Paris (http://comitedufilmethnographique.com) et chercheur associé à l'IREMAM, CNRS-MMSH Aix-en-Provence. Après avoir été chargé de mission à la Mission du Patrimoine ethnologique (Gard) et enseigné l'ethnologie à l'Université de Montpellier III, il est aujourd'hui maître de conférences en anthropologie visuelle et filmique au département des Arts du spectacle de l'Université de Nanterre où il enseigne l'histoire, la théorie et la pratique du cinéma en anthropologie. Ses travaux portent sur le cinéma documentaire, l'anthropologie visuelle et filmique, le milieu ouvrier, le champ de la santé, de la médecine et de la maladie. Il est également membre du comité de rédaction de la revue Anthropologie \& Santé et porteur du projet Labex Arts-H2H : Consultation médicale virtuelle (CMV) : http://www.labex-arts-h2h.fr/ consultation-medicale-virtuelle.html 